\title{
Resistance to the c-Met inhibitor KRC-108 induces the epithelial transition of gastric cancer cells
}

\author{
DONG CHUL KIM ${ }^{1 *}$, KYEONG RYANG PARK ${ }^{2,3^{*}}$, YEON JI JEONG ${ }^{2,3}$, HYONOK YOON $^{2,3}$, MI-JEONG AHN $^{2,3}$, \\ GYU-JIN RHO ${ }^{4}$, JONGKOOK LEE ${ }^{5}$, YOUNG-DAE GONG ${ }^{6}$ and SUN-YOUNG HAN ${ }^{2,3}$ \\ ${ }^{1}$ Department of Pathology, School of Medicine; ${ }^{2}$ College of Pharmacy; ${ }^{3}$ Research Institute of Life Sciences; \\ ${ }^{4}$ Department of Theriogenology and Biotechnology, School of Veterinary Medicine, Gyeongsang National University, \\ Jinju, Gyeongsang 52828; ${ }^{5}$ College of Pharmacy, Kangwon National University, Chuncheon, Gangwon 200-701; \\ ${ }^{6}$ Innovative Drug Library Research Center, Dongguk University, Seoul 100-715, Republic of Korea
}

Received October 30, 2014; Accepted August 17, 2015

DOI: $10.3892 / \mathrm{ol} .2015 .4029$

\begin{abstract}
Investigation of the mechanisms of resistance to targeted therapies is essential as resistance acquired during treatment may lead to relapse or refractoriness to the therapy. Our previous study identified the small molecule KRC-108 as a result of efforts to find an anticancer agent with c-Met-inhibitory activity. In the present study, the changes accompanying resistance to KRC-108 were investigated in the gastric cancer cell line MKN-45 and its KRC-108-resistant clones by western blot and immunofluorescence analyses. Increased expression of the c-Met protein was observed in KRC-108-resistant cells compared with that of the parental cells, and the phosphorylation of c-Met also increased in cell lines resistant to KRC-108. Resistance to the c-Met inhibitor was associated with cell morphological changes: MKN-45 parental cells, which had a round and poorly differentiated morphology, were altered to exhibit an epithelial cell-like phenotype in KRC-108-resistant clones. Consistent with the transition to an epithelial morphology, the expression of E-cadherin was increased in resistant cells. Using immunoprecipitation, an interaction between E-cadherin and the c-Met protein was observed in the KRC-108-resistant cells. Immunohistochemical analysis of
\end{abstract}

Correspondence to: Dr Sun-Young Han, College of Pharmacy, Gyeongsang National University, 501 Jinju-daero, Jinju, Gyeongsang 52828, Republic of Korea

E-mail: syhan@gnu.ac.kr

*Contributed equally

Abbreviations: HGF, hepatocyte growth factor; NSCLC, non-small-cell lung cancer; EGFR, epidermal growth factor receptor; EMT, epithelial-mesenchymal transition; MET, mesenchymal-epithelial transition; GIST, gastrointestinal stromal tumor; $\mathrm{GI}_{50}$, growth inhibition of 50\%; TBST, Tris-buffered saline containing $0.1 \%$ Tween-20; PBS, phosphate-buffered saline; BSA, bovine serum albumin; RT, room temperature

Key words: c-Met, E-cadherin, drug resistance, epithelial transition human gastric carcinoma tissues revealed the co-expression of E-cadherin and c-Met. These results suggest that the epithelial transition in KRC-108-resistant cells is mediated by recruiting E-cadherin to c-Met protein. Thus, the present study identified a mechanism used by cancer cells to confer resistance to anticancer agents.

\section{Introduction}

Gastric cancer accounts for $7.8 \%$ of cancers worldwide (1). Furthermore, it is the fourth most common type of cancer, and the second most common cause of cancer-related mortality worldwide $(2,3)$. Approximately $85 \%$ of stomach cancers are adenocarcinomas (4). The gastric antrum is the most common site of gastric carcinoma (1). Endoscopy is useful for diagnosis as it exhibits a high level of sensitivity and specificity (1). Gastric carcinomas are usually asymptomatic in the early stages of the disease (4). However, as the carcinomas progress, symptoms including, upper abdominal discomfort, anorexia, nausea, vomiting and weight loss may develop. Gastric carcinomas may metastasize to lymph nodes, perigastric tissue, pancreas, colon, liver and the ovaries (4). Gastric cancer treatment and prognosis are dependent on tumor stage (3). Endoscopic mucosal resection may be used for the treatment of early stage gastric carcinoma lesions, $<2 \mathrm{~cm}$ in size, without invasion of the lamina propria or muscularis mucosa (3). Complete resection of tumors by subtotal gastrectomy or total gastrectomy with lymph node dissection are the first choices for curative treatment. However, surgery is applicable in less than a third of patients (4). In advanced gastric cancers, additional chemotherapy or chemoradiation treatment prior to or following surgery is considered a good option $(2,3)$. It has been found that overall survival is increased by $9 \%$ after neoadjuvant chemotherapy (3). Furthermore, adjuvant chemotherapy with various regimens, including 5-fluorouracil, have been found to increase overall survival by $6 \%$ and reduce the risk of mortality by $18 \%$ (3). Targeted therapies involving the inhibition of human epidermal growth factor receptor 2, vascular endothelial growth factor receptor 2 and epidermal growth factor receptor (EGFR) have also been investigated $(2,3)$. Despite advances in treatment, the survival of advanced 
gastric carcinoma patients remains poor with a 5-year survival rate of $<30 \%$ in stage III patients $(1,3,4)$. Response rates for various chemotherapies range between 9 and $51 \%$ (5). However,these response rates do not correlate with survival rate. The discrepancy is associated with drug resistance, which leads to the failure of therapy and poor prognosis. At present, the mechanisms of anticancer drug resistance remain unclear, despite extensive investigation.

Anticancer agents targeting specific proteins in cancer cells have revolutionized pharmacotherapy in the field of oncology (6). However, targeted therapies have limitations due to the development of resistance with long-term use, in which certain types of cancer cells acquire resistance to drugs that inhibit specific proteins (7). Resistance to anticancer drugs results in relapse or refractoriness to therapy for cancer patients (7). Thus, during the development of targeted agents, the resistance mechanisms must be investigated in order to aid in overcoming the problems associated with such resistance.

Inhibitors of c-Met kinase have been investigated as anticancer agents and subjected to clinical trials (8). The receptor tyrosine kinase c-Met is activated by its ligand, hepatocyte growth factor (HGF). Upon binding to HGF, c-Met dimerizes and transduces cell signaling by activating multiple downstream pathways, including Akt, mitogen-activated protein kinase and focal adhesion kinase. The physiological roles of the c-Met signaling pathway include cell survival and migration; therefore, dysregulated c-Met activation may lead to tumorigenesis (8). Mutation or overexpression of the c-Met protein has been reported in various types of cancer, including gastric cancer $(9,10)$. Blocking c-Met activity using small molecule inhibitors or monoclonal antibodies may be an effective strategy for cancer therapy, and numerous c-Met inhibitors are currently under development for anticancer drugs (11). Among the drugs approved by the Food and Drug Administration, cabozantinib (also known as XL-184) is an inhibitor of c-Met, vascular endothelial growth factor receptor 2, and Ret proto-oncogene and is used for treatment of medullary thyroid cancer $(12,13)$.

$\mathrm{KRC}-108$ is a small molecule that was previously identified in an effort to find inhibitors of c-Met with oral antitumor properties (14). In order to identify the mechanisms underlying resistance to $\mathrm{KRC}-108$ treatment, the present study generated gastric cancer cell lines resistant to KRC-108, and the phenotypic and molecular changes associated with KRC-108 resistance were investigated.

\section{Materials and methods}

Generation of KRC-108-resistant cell lines. MKN-45 human gastric cancer cells were purchased from the Korean Cell Line Bank (Seoul, Korea) and cultured in RPMI-1640 medium (GE Healthcare Life Sciences HyClone Laboratories, Logan, UT, USA) supplemented with $10 \%$ fetal bovine serum (GE Healthcare Life Sciences HyClone Laboratories). The cells were incubated at $37^{\circ} \mathrm{C}$ in a humidified atmosphere of $5 \%$ $\mathrm{CO}_{2}$. KRC-108-resistant clones of MKN-45 cell lines were established by exposing the cells to increasing concentrations of KRC-108 (synthesized by Professor Jongkook Lee; Kangwon National University, Kangwon, South Korea) for 3 months. The cells were grown in the presence of KRC-108
Table I. Expression of E-cadherin and c-Met in human gastric carcinoma tissues.

\begin{tabular}{lrrrr}
\hline & \multicolumn{3}{c}{ c-Met expression, $\mathrm{n}$} \\
\cline { 2 - 3 } E-cadherin expression & \multicolumn{1}{c}{+} & + & ++ & Total \\
\hline- & 3 & 0 & 0 & 3 \\
+ & 8 & 5 & 0 & 13 \\
++ & 12 & 18 & 25 & 55 \\
Total & 23 & 23 & 25 & 71 \\
\hline
\end{tabular}

Tissue microarray blocks of gastric carcinoma tissues were subjected to immunohistochemical staining using antibodies against E-cadherin and c-Met, respectively. Staining results were categorized into three groups according to intensity: -, negative; +, medium intensity; ++, strong intensity. The number of samples in each group is indicated in the table.

at $100 \mathrm{nM}$ at first for two weeks, and the concentration of KRC-108 was increased gradually to a final concentration of $1 \mu \mathrm{M}$. Replicate clones capable of proliferating in $1 \mu \mathrm{M}$ of KRC-108 were used in this study.

Cell viability assay. The cells were plated in a 96-well plate (2,000 cells/well), and serial dilutions of KRC-108 were added. At the end of the incubation period ( $72 \mathrm{~h}$ ), cell viability was measured using the tetrazolium-based EZ-CYTOX cell viability assay kit (DaeilLab Service Co., Ltd., Seoul, Korea). Growth inhibition of $50 \%\left(\mathrm{GI}_{50}\right)$ was calculated by non-linear regression using GraphPad Prism software version 5.01 (GraphPad Software, Inc., La Jolla, CA, USA).

Western blot analysis. Samples of the cell extracts prepared in sodium dodecyl sulfate (SDS) lysis buffer (12 mM Tris- $\mathrm{Cl}, \mathrm{pH}$ 6.8; 5\% glycerol; $0.4 \%$ SDS; USB Corporation, Cleveland, OH, USA) were resolved by SDS-PAGE and transferred to a polyvinylidene fluoride membrane (EMD Millipore, Billerica, MA, USA). The filters were blocked in Tris-buffered saline (10 mM Tris-Cl, pH 7.4; $140 \mathrm{mM} \mathrm{NaCl}$ ) containing $0.1 \%$ Tween-20 (TBST) and 5\% non-fat dry milk. Subsequently, the filters were incubated with a blocking solution containing the indicated primary antibodies for $2 \mathrm{~h}$. After washing three times in TBST, the membranes were incubated with a peroxidase-conjugated secondary antibody for $1 \mathrm{~h}$ at room temperature (RT). Blots were then washed three times and developed using chemiluminescent substrate (GE Healthcare Life Sciences, Chalfont, UK), and luminescent signals were visualized using an ImageQuant LAS 4000 Mini (GE Healthcare Life Sciences). Primary antibodies against human c-Met (rabbit polyclonal IgG; \#sc-10; 1:1,000), phosphorylated (p-)Met (rabbit polyclonal IgG; \#sc-34085; 1:1,000), E-cadherin (mouse monoclonal IgG; \#sc-21791; 1:1,000), N-cadherin (rabbit polyclonal IgG; \#sc-7939; 1:1,000; all from Santa Cruz Biotechnology, Inc., Dallas, TX, USA) and $\beta$-actin (mouse monoclonal IgG; \#A5441; 1:5,000; Sigma-Aldrich, St. Louis, MO, USA) were used. Goat anti-rabbit IgG (\#111-035-003; 1:5,000) and anti-mouse IgG (\#115-035-003; 1:5,000) secondary antibodies were 


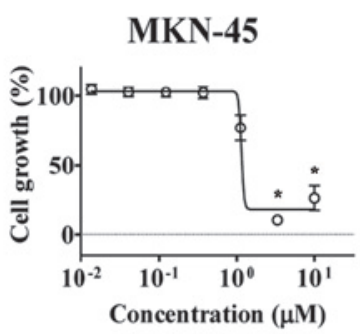

B

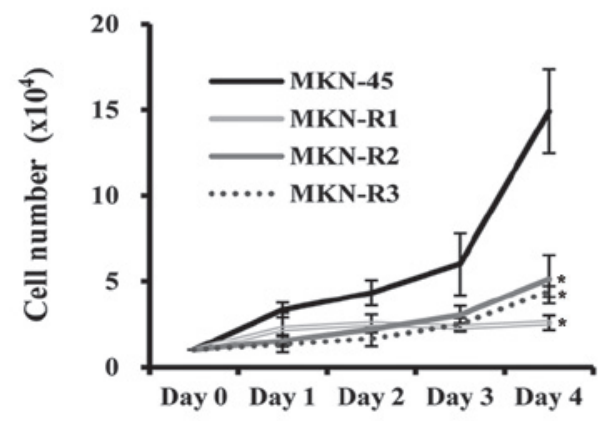

D
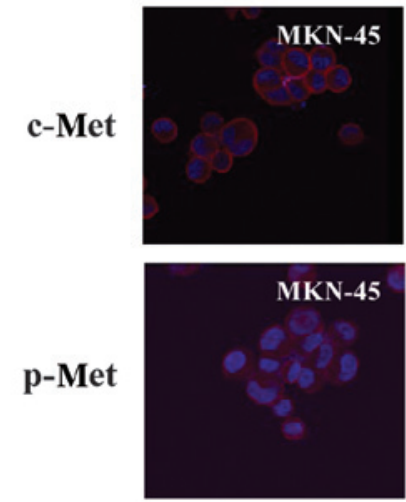

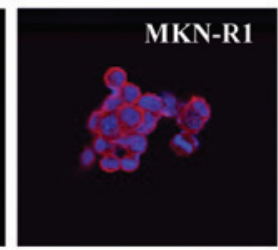

MKN-R1
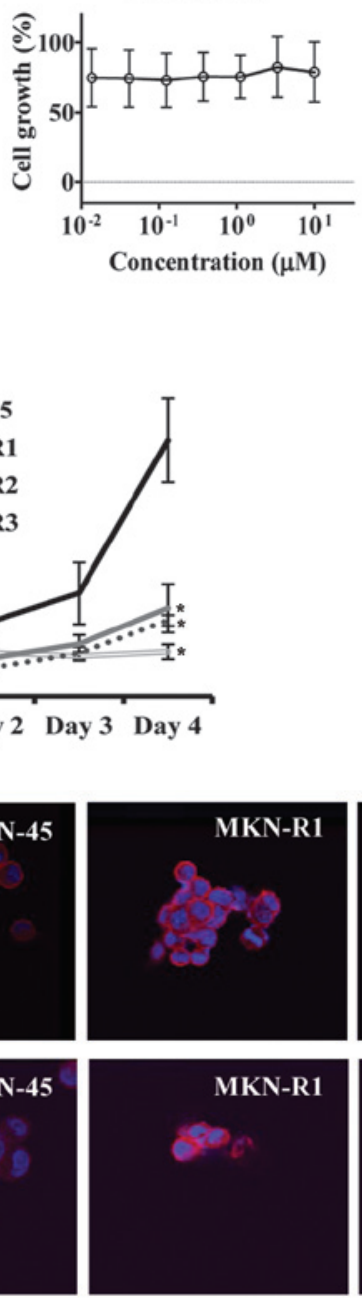

MKN-R2



C

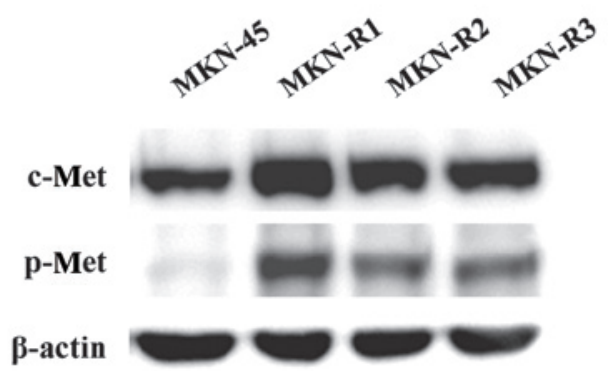

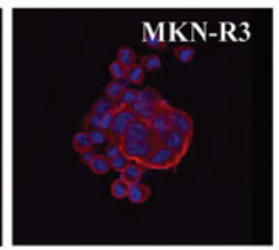


Figure 1. Characteristics of MKN-45 human gastric cancer cells and KRC-108-resistant clones (MKN-R1, -R2 and -R3). (A) MKN-45, MKN-R1, MKN-R2 and MKN-R3 cells were treated with KRC-108 at the indicated concentrations for $72 \mathrm{~h}$ before subjection to a cell viability assay. Data are presented as the means from three independent experiments and bars represent standard error. ${ }^{*} \mathrm{P}<0.05$ vs. DMSO control. (B) Cells were seeded into a 24 -well plate, and the cell numbers were counted each day until day 4 to establish a growth curve. Data are presented as the mean and standard error of three independent experiments. ${ }^{*} \mathrm{P}<0.05$ vs. MKN-45 cells (C) The expression levels of c-Met and p-Met following KRC-108 treatment were measured by western blotting in MKN-45 and MKN-R cells. (D) The expression of c-Met and the phosphorylation of c-Met were analyzed by immunofluorescence (magnification, x630). Red, c-Met (top row) or p-Met (bottom row); blue, DAPI. p-Met, phosphorylated c-Met.

purchased from Jackson ImmunoResearch Laboratories, Inc. (West Grove, PA, USA).

Immunoprecipitation. Cell lysates were prepared in NETN lysis buffer [0.5\% NP-40 (Abcam, Cambridge, MA, USA), $1 \mathrm{mM}$ EDTA (Bioneer, Daejeon, South Korea), $120 \mathrm{mM} \mathrm{NaCl}$, $1 \mathrm{mM}$ DTT (Sigma-Aldrich), $10 \mathrm{mM} \mathrm{NaF}$ (Sigma-Aldrich), $2 \mathrm{mM} \mathrm{Na} \mathrm{VO}_{4}$ (Sigma-Aldrich), $50 \mathrm{mM}$ Tris-Cl, $\mathrm{pH}$ 8.0) with a protease inhibitor cocktail (Sigma-Aldrich). Lysates were incubated with the indicated antibodies overnight at $4^{\circ} \mathrm{C}$ and Protein G Sepharose beads (GE Healthcare Life Sciences) were added. Following incubation for $2 \mathrm{~h}$, the immune complexes were washed and released from the beads by boiling and then analyzed by western blotting using the indicated antibodies.

Immunofluorescence. The cells were plated on 8-chamber culture slides and incubated for $24 \mathrm{~h}$. They were then rinsed briefly in phosphate-buffered saline (PBS), fixed in $4 \%$ formaldehyde (Sigma-Aldrich) and subsequently permeabilized in
$0.2 \%$ Triton X-100 (Sigma-Aldrich). After washing the samples twice with ice-cold PBS, the cells were blocked with $1 \%$ bovine serum albumin (BSA; Gibco; Thermo Fisher Scientific, Waltham, MA, USA) in PBS with Tween-20 (PBST; Anatrace Inc., Maumee, OH, USA) for $30 \mathrm{~min}$ and then incubated with the primary antibody (1:50) (diluted in 1\% BSA in PBST) overnight at RT. Following three washes with PBS, the cells were incubated with the corresponding secondary antibody [Alexa Fluor $^{\circledR} 488$ goat anti-mouse IgG (H+L) (\#A-11001; 1:500) or Alexa Fluor ${ }^{\circledR} 546$ goat anti-rabbit IgG (H+L) (\#A-11010; 1:500); Invitrogen Life Technologies, Carlsbad, CA, USA] for $4 \mathrm{~h}$ at RT in the dark. Mounting medium (ProLong ${ }^{\circledR}$ Gold Antifade reagent with DAPI; Invitrogen Life Technologies) was dropped onto the washed cells, which were subsequently photographed with a confocal laser scanning microscope (FV-1000; Olympus Corporation, Tokyo, Japan).

Tissue microarray. Tumor samples from 71 patients with primary gastric cancer that had undergone laparoscopic 

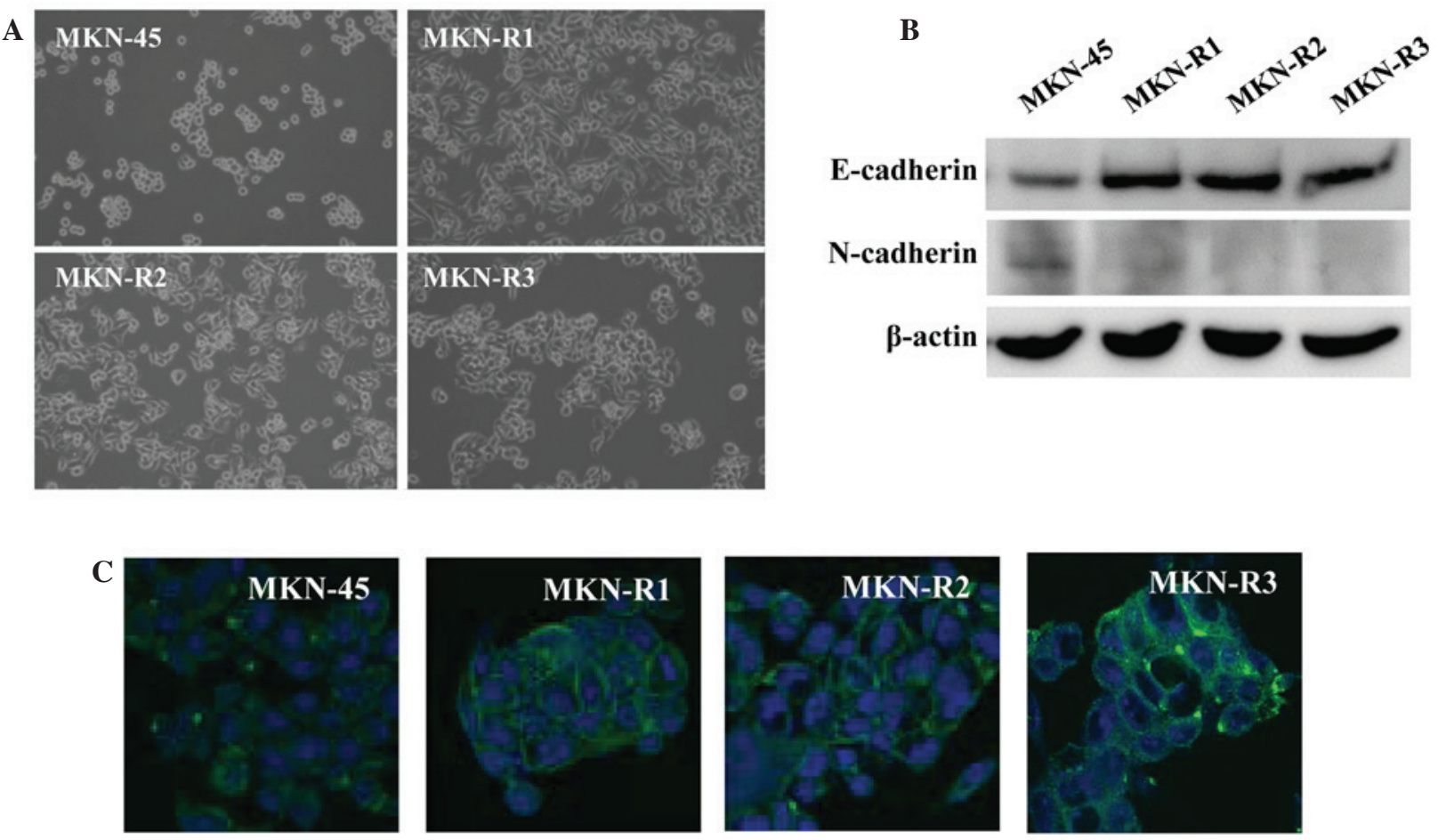

Figure 2. Epithelial transition of MKN-45 cell line and clones resistant to KRC-108 (MKN-R1, -R2 and R3). (A) Phase contrast images of the MKN-45 and MKN-R cells (magnification, x100). (B) The expression of E-cadherin and N-cadherin in the MKN-45 and MKN-R cells was detected by western blotting. (C) E-cadherin expression was analyzed by immunofluorescence: Green, E-cadherin; blue, DAPI (magnification, x630).

distal gastrectomy, subtotal gastrectomy or total gastrectomy at Gyeongsang National University Hospital (Jinju, Korea) in 2010 were collected. The patient cohort included 53 males and 18 females, with a mean age of 63.5 years (range, 36-80 years). The pathologist reviewed hematoxylin and eosin-stained slides from neutral buffered formalin-fixed, paraffin-embedded tissue blocks. One representative area of carcinoma was selected from each case. Three tissue microarray blocks containing 71 cores of $3 \mathrm{mM}$ diameter were constructed. The process was conducted using a precision arraying instrument (Quick Ray ${ }^{\circledR}$; Unitma Co., Ltd., Seoul, Korea). This study was approved by the Institutional Review Board (IRB) of Gyeongsang National University Hospital and a waiver of written informed consent was obtained.

Immunohistochemistry. Immunohistochemical staining of the tumor specimens was conducted using a Benchmark XT autostainer (Ventana Medical Systems, Inc., Tucson, AZ, USA) using anti-E-cadherin and anti-c-Met antibodies. E-cadherin staining was assessed and cases were divided into three groups according to membrane staining: -, + or ++ . Cases with no staining or partial cell membrane staining were interpreted as negative (-). Cases with discontinuous staining along the cell membrane were interpreted as positive $(+)$ and cases stained continuously along the cell membrane were interpreted as strongly positive (++). c-Met was interpreted with regard to cytoplasmic staining, with positivity classified into three groups according to intensity; -, + or ++. Cases exhibiting no staining were interpreted as negative (-), cases exhibiting partial staining were interpreted as positive $(+)$ and cases exhibiting complete cytoplasmic staining were interpreted as strongly positive (++).



Figure 3. Interaction of c-Met and E-cadherin in MKN-45 human gastric cancer cells and KRC-108-resistant clones (MKN-R1, -R2 and -R3). Cell lysates were immunoprecipitated with a c-Met antibody, and the immunoprecipitates were probed with c-Met and E-cadherin antibodies.

Statsitical analysis. Data were analyzed using SPSS software version 18.0 (SPSS, Inc., Chicago, IL, USA). Correlations were assessed using the $\chi^{2}$ test. $\mathrm{P}<0.05$ was considered to indicate a statistically significant difference.

\section{Results}

Establishment of MKN-45 cells resistant to c-Met kinase inhibitor KRC-108. The KRC-108 compound is an antitumor agent with c-Met inhibitory activity in vitro and in vivo (14). To study the mechanisms associated with acquired resistance 

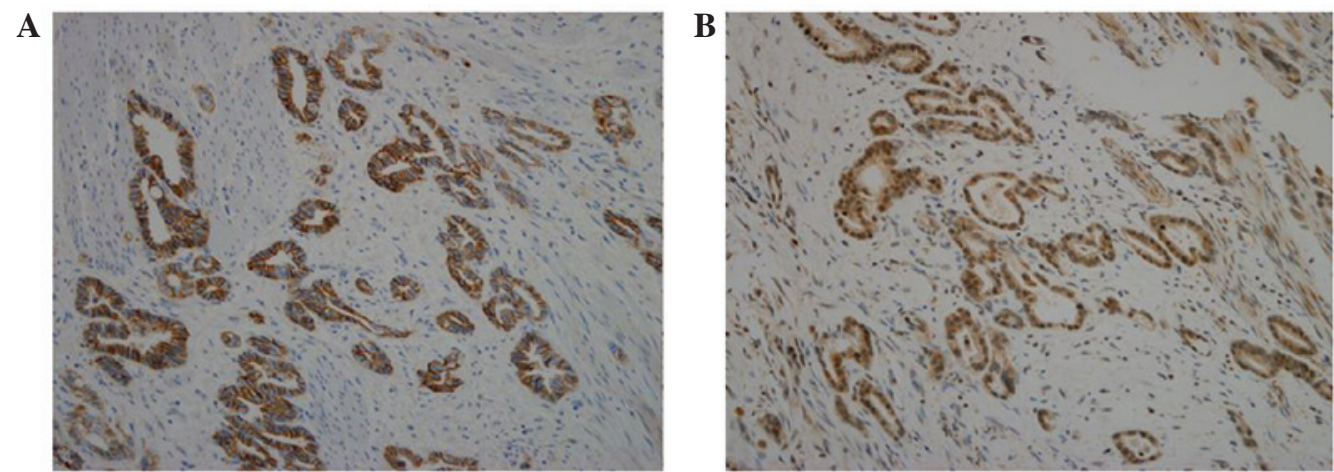

Figure 4. Immunohistochemical analysis of human gastric carcinoma tissues. (A) E-cadherin staining and (B) c-Met staining. Representative images of samples with strong intensity are shown (magnification, x200).

to KRC-108, the gastric cancer cell line MKN-45, which expresses a high level of c-Met (15), was utilized to develop KRC-108-resistant cell lines. The cells were treated with KRC-108, initially at a low concentration, and the dose was increased stepwise. The resulting KRC-108-resistant cells were designated MKN-R, and three replicate clones were used: MKN-R1, MKN-R2, and MKN-R3. The parental MKN-45 cells were sensitive to KRC-108 treatment with the $\mathrm{GI}_{50}$ concentration of $1.1 \mu \mathrm{M}$, whilst the MKN-R cells did not exhibit growth inhibition with treatment of KRC-108 up to a concentration of $10 \mu \mathrm{M}$ (Fig. 1A). The growth characteristics of the MKN-45 and MKN-R cells were different, with the MKN-R cells growing more slowly than the parental MKN-45 cells, as shown in Fig. 1B. Western blot analysis was conducted to investigate the effect of KRC-108 treatment, revealing increased expression of c-Met in the MKN-R cells compared with that of the parental cells (Fig. 1C). Along with the overexpression of c-Met, the phosphorylated form of c-Met (p-Met) was increased in the MKN-R cells compared with the parental MKN-45 cells. The increase in the expression level and the activity of c-Met was confirmed by immunofluorescence (Fig. 1D). We hypothesized that a high level of active c-Met (p-Met) may cause the MKN-R cells to be resistant to KRC-108 treatment. The inhibition of c-Met kinase activity by KRC-108 was overcome by overexpression of the c-Met protein, thus resulting in cell survival in the presence of KRC-108.

KRC-108-resistant cells have an epithelial cell-like phenotype. A phenotypic difference was observed between the MKN-45 cells and the MKN-R cells. Fig. 2A displays the phase contrast images of the cells, demonstrating morphological changes in the KRC-108-resistant cells. The parental MKN-45 cells were round with a poorly differentiated form, whilst the MKN-R cells exhibited a flat epithelial cell-like phenotype. All three clones of MKN-R cells displayed similar morphology.

Consistent with the epithelial characteristics of the MKN-R cells, higher expression of E-cadherin in MKN-R cells relative to the MKN-45 cells was observed (Fig. 2B). E-cadherin is an epithelial marker and cell-surface adhesion protein $(16,17)$. In addition, expression levels of $\mathrm{N}$-cadherin, a mesenchymal marker, were decreased in MKN-R cells relative to MKN-45 cells. To confirm the change in the expression of E-cadherin observed on the western blot, immunostaining using an anti-E-cadherin antibody was performed. Immunocytochemical analyses of E-cadherin revealed high expression of E-cadherin in the cell surface area of the MKN-R cells (Fig. 2C). These results indicate that E-cadherin is expressed in cell-cell contact areas of MKN-R cells.

c-Met associates with E-cadherin in $M K N-R$ cells. Next, the mechanism of morphological change and upregulation of E-cadherin expression associated with KRC-108 resistance were investigated. As a direct interaction between c-Met and E-cadherin has been reported in a number of studies (18-20), the possibility of direct binding of c-Met and E-cadherin in the MKN-R cells was explored using immunoprecipitation. Cell lysates were immunoprecipitated with an anti-c-Met antibody, and the immunoprecipitates were subjected to SDS-PAGE and probed with an anti-E-cadherin antibody. As shown in Fig. 3, E-cadherin was detected in the immunoprecipitates from the MKN-R cells. Thus, c-Met and E-cadherin interacted in the MKN-R cells, but not in the MKN-45 cells. These results imply that the epithelial transition in the MKN-R cells is mediated by overexpression of c-Met, leading to the recruitment of E-cadherin to the cell surface. The recruitment of E-cadherin by c-Met may induce epithelial cell-like changes in MKN-R cells.

Double expression of E-cadherin and c-Met in human gastric cancer tissues. To confirm the association of c-Met and E-cadherin in human tissue samples, a tissue microarray was performed using specimens of human gastric carcinoma. Tissue microarray blocks were subjected to immunohistochemical staining using antibodies against E-cadherin and c-Met. As shown in Table I, 48 of the 71 cores (67.6\%) exhibited double expression of E-cadherin and c-Met. Furthermore, all samples with c-Met ++ expression exhibited high expression of E-cadherin (++). As shown in Fig. 4, staining for E-cadherin and c-Met revealed the same pattern of expression in gastric carcinoma. The co-expression of E-cadherin and c-Met observed in human gastric carcinoma tissues supports the findings of an association of c-Met with E-cadherin in gastric carcinoma cell lines (Fig. 3).

\section{Discussion}

The present study explored the cellular changes accompanied by resistance to the c-Met inhibitor KRC-108 and their possible 
mechanisms. The results revealed an overexpression of c-Met and phenotypic changes to more epithelial characteristics in MKN-R cells. Mechanisms of resistance to other c-Met inhibitors have been reported in previous studies (21-23). McDermott et al (21) investigated drug resistance to a c-Met inhibitor, PF-2341066, in non-small-cell lung cancer (NSCLC) cell lines. The study utilized NSCLC cells with c-Met amplification, which were sensitive to c-Met inhibitors. Prolonged exposure to c-Met inhibitor PF-2341066 resulted in activation of EGFR in the cells. Resistant cells with EGFR activation demonstrated sensitivity to combined treatment with PF-2341066 and an EGFR inhibitor, suggesting that the EGFR pathway compensated for c-Met signaling in resistant cells. A similar mechanism was reported in the gastric cancer cell line SNU638 (22). The c-Met inhibitors PHA-665752 and PF-2341066 were utilized to develop c-Met-resistant SNU638 cells. Two mechanisms of resistance were revealed: One involving the same mechanism observed in NSCLC cells (i.e., activation of EGFR), and the other involving a mutation of the c-Met sequence, Y1230C. Y1230 is located in the activation loop of c-Met, and its mutation results in structural changes that affect interaction with c-Met inhibitors. In a separate study, a drug resistance screen using other c-Met inhibitors, NVP-BYU972 and AMG458, also demonstrated mutations in the c-Met sequence affecting binding of c-Met inhibitors (23). In the current paper, the mechanism of $\mathrm{KRC}-108$ resistance was the overexpression of c-Met. Overexpression of the target protein is a frequently observed mechanism of drug resistance, as demonstrated by Bcr-Abl inhibitors (7). Some of leukemic cells were found to develop resistance to Bcr-Abl inhibitor imatinib following a few years of use in leukemic patients. Several mechanisms of resistance were revealed and amplification of the $B C R-A B L$ gene and subsequent overexpression of the Bcr-Abl kinase was frequently observed (24).

The epithelial-mesenchymal transition (EMT) is known to be crucial in cancer progression (25). The EMT involves phenotypic changes from an epithelial to mesenchymal cell type, with the acquisition of migratory and invasive properties. The EMT process is reversible, and the reverse process is termed the mesenchymal-epithelial transition (MET). The term 'epithelial-mesenchymal plasticity' was previously proposed to describe the flexible transitions observed between epithelial cells and mesenchymal cells (26). The changes observed in the MKN-R cells in the present study were similar to those seen in MET. The MKN-R cells acquired epithelial morphology, which was accompanied by upregulation of E-cadherin (Fig. 2). We hypothesize that epithelial plasticity may be closely related to drug resistance in cancer cells.

Morphological changes, such as the EMT/MET phenomena, accompanied by drug resistance have been described in a number of studies (27-30). Many reported a positive correlation between EMT activation and drug resistance (27-29). EMT was observed in lung cancer cells with EGFR inhibitor resistance (28). Thomson et al (29) reported that cells insensitive to the EGFR inhibitor have a mesenchymal phenotype. By contrast, another report revealed that drug-resistant cell lines exhibited epithelial cell characteristics (30). The study by Mahadevan et al (30) of gastrointestinal stromal tumor (GIST) cells resistant to imatinib reported that imatinib-resistant GIST cells changed to an epithelial cell-like phenotype. From the available literature and the results of the present study, it is not clear whether the EMT confers drug resistance. However, it is clear that cellular plasticity represented by morphological changes is frequently observed in drug-resistant cells and thus, we hypothesize that such cells lose or acquire epithelial characteristics. The direction of change (EMT or MET) may be dependent on the specific cell types used in the experiments.

Various studies of the association of c-Met with E-cadherin in a number of tumor cell lines have been published (18-20,31). The co-localization of c-Met and $\beta$-catenin in a cell adhesion complex, as well as E-cadherin, was observed in breast and colorectal cancer cell lines (18). Forced expression of E-cadherin in BT-549 human breast cancer cells, which do not express E-cadherin, resulted in the recruitment of c-Met to the cell membrane (19). A tissue microarray study of ductal breast carcinoma in situ revealed a correlation between c-Met and E-cadherin expression (20). The importance of the c-Met/E-cadherin interaction has also been demonstrated in pathological states other than cancer: Helicobacter pylori infection induced an invasive phenotype in gastric epithelial cells (31), and E-cadherin expression suppressed the invasive phenotype through an interaction with c-Met. In the present study, the interaction of c-Met and E-cadherin is presumed to have contributed to the epithelial phenotype in the MKN-R cells (Fig. 3), consistent with previous reports.

In conclusion, the present study revealed a mechanism utilized by cancer cells to confer resistance to anticancer agents. These results may aid the establishment of therapeutic strategies in cases of c-Met kinase inhibitor resistance. The association between tumor morphology and c-Met inhibitor resistance requires further study. Accumulating knowledge of resistance mechanisms will aid the development of successful therapy with targeted agents in the future.

\section{Acknowledgements}

This work was supported by grants of the National Research Foundation (2015R1C1A2A01053928) funded by the government of Korea, and grants from the Ministry of Trade, Industry \& Energy and Korea Institute for Advancement of Technology through Inter-Economic Region co-operation projects (A004500005).

\section{References}

1. Bosman FT, Carneiro F, Hruban RH and Theise ND (eds): WHO Classification of Tumours of the Digestive System. 4th edition. IARC Press, Lyon, 2010.

2. Carcas LP: Gastric cancer review. J Carcinog 13: 14, 2014.

3. Marrelli D, Polom K, de Manzoni G, Morgagni P, Baiocchi GL and Roviello F: Multimodal treatment of gastric cancer in the west: Where are we going? World J Gastroenterol 21: 7954-7969, 2015.

4. Kasper DL, Fauci A, Hauser S, Longo D, Jameson J and Loscalzo J (eds): Harrison's Principles of Internal Medicine. 19th edition. McGraw-Hill Education, New York, NY, 2015.

5. Koizumi W: Chemotherapy for advanced gastric cancer: Review of global and Japanese status. Gastrointest Cancer Res 1: 197-203, 2007.

6. Aggarwal S: Targeted cancer therapies. Nat Rev Drug Discov 9: 427-428, 2010.

7. Engelman JA and Settleman J: Acquired resistance to tyrosine kinase inhibitors during cancer therapy. Curr Opin Genet Dev 18: 73-79, 2008 
8. Gherardi E, Birchmeier W, Birchmeier C and Vande Woude G: Targeting MET in cancer: Rationale and progress. Nat Rev Cancer 12: 89-103, 2012.

9. Kuniyasu H, Yasui W, Kitadai Y, Yokozaki H, Ito H and Tahara E: Frequent amplification of the c-met gene in scirrhous type stomach cancer. Biochem Biophys Res Commun 189: 227-232, 1992.

10. Hara T, Ooi A, Kobayashi M, Mai M, Yanagihara K and Nakanishi I: Amplification of c-myc, K-sam and c-met in gastric cancers: Detection by fluorescence in situ hybridization. Lab Invest 78: 1143-1153, 1998 .

11. Scagliotti GV, Novello S and von Pawel J: The emerging role of MET/HGF inhibitors in oncology. Cancer Treat Rev 39: 793-801, 2013.

12. Zhang Y, Guessous F, Kofman A, Schiff D and Abounader R: XL-184, a MET, VEGFR-2 and RET kinase inhibitor for the treatment of thyroid cancer, glioblastoma multiforme and NSCLC. IDrugs 13: 112-121, 2010.

13. Hoy SM: Cabozantinib: A review of its use in patients with medullary thyroid cancer. Drugs 74: 1435-1444, 2014.

14. Han SY, Lee CO, Ahn SH, Lee MO, Kang SY, Cha HJ, Cho SY, Ha JD, Ryu JW, Jung H, et al: Evaluation of a multi-kinase inhibitor KRC-108 as an anti-tumor agent in vitro and in vivo. Invest New Drugs 30: 518-523, 2012.

15. McDermott U, Sharma SV, Dowell L, Greninger P, Montagut C, Lamb J, Archibald H, Raudales R, Tam A, Lee D, et al: Identification of genotype-correlated sensitivity to selective kinase inhibitors by using high-throughput tumor cell line profiling. Proc Natl Acad Sci USA 104: 19936-19941, 2007.

16. Gumbiner BM: Cell adhesion: The molecular basis of tissue architecture and morphogenesis. Cell 84: 345-357, 1996.

17. Takeichi M: Cadherin cell adhesion receptors as a morphogenetic regulator. Science 251: 1451-1455, 1991.

18. Hiscox S and Jiang WG: Association of the HGF/SF receptor, c-met, with the cell-surface adhesion molecule, E-cadherin and catenins in human tumor cells. Biochem Biophys Res Commun 261: 406-411, 1999.

19. Reshetnikova G, Troyanovsky S and Rimm DL: Definition of a direct extracellular interaction between Met and E-cadherin. Cell Biol Int 31: 366-373, 2007.

20. Götte M, Kersting C, Radke I, Kiesel L and Wülfing P: An expression signature of syndecan-1 (CD138), E-cadherin and c-met is associated with factors of angiogenesis and lymphangiogenesis in ductal breast carcinoma in situ. Breast Cancer Res 9: R8, 2007.

21. McDermott U, Pusapati RV, Christensen JG, Gray NS and Settleman J: Acquired resistance of non-small cell lung cancer cells to MET kinase inhibition is mediated by a switch to epidermal growth factor receptor dependency. Cancer Res 70: $1625-1634,2010$.
22. Qi J, McTigue MA, Rogers A, Lifshits E, Christensen JG, Jänne PA and Engelman JA: Multiple mutations and bypass mechanisms can contribute to development of acquired resistance to MET inhibitors. Cancer Res 71: 1081-1091, 2011.

23. Tiedt R, Degenkolbe E, Furet P, Appleton BA, Wagner S, Schoepfer J, Buck E, Ruddy DA, Monahan JE, Jones MD, et al: A drug resistance screen using a selective met inhibitor reveals a spectrum of mutations that partially overlap with activating mutations found in cancer patients. Cancer Res 71: 5255-5264, 2011.

24. Gorre ME, Mohammed M, Ellwood K, Hsu N, Paquette R, Rao PN and Sawyers CL: Clinical resistance to STI-571 cancer therapy caused by BCR-ABL gene mutation or amplification. Science 293: 876-880, 2001.

25. Hugo H, Ackland ML, Blick T, Lawrence MG, Clements JA, Williams ED and Thompson EW: Epithelial-mesenchymal and mesenchymal-epithelial transitions in carcinoma progression. J Cell Physiol 213: 374-383, 2007.

26. Thompson EW and Haviv I: The social aspects of EMT-MET plasticity. Nat Med 17: 1048-1049, 2011.

27. Tsai $\mathrm{JH}$ and Yang J: Epithelial-mesenchymal plasticity in carcinoma metastasis. Genes Dev 27: 2192-2206, 2013.

28. Xie M, Zhang L, He CS, Xu F, Liu JL, Hu ZH, Zhao LP and Tian Y: Activation of Notch-1 enhances epithelial-mesenchymal transition in gefitinib-acquired resistant lung cancer cells. J Cell Biochem 113: 1501-1513, 2012.

29. Thomson S, Buck E, Petti F, Griffin G, Brown E, Ramnarine N, Iwata KK, Gibson N and Haley JD: Epithelial to mesenchymal transition is a determinant of sensitivity of non-small-cell lung carcinoma cell lines and xenografts to epidermal growth factor receptor inhibition. Cancer Res 65: 9455-9462, 2005.

30. Mahadevan D, Cooke L, Riley C, Swart R, Simons B, Della Croce K, Wisner L, Iorio M, Shakalya K, Garewal H, et al: A novel tyrosine kinase switch is a mechanism of imatinib resistance in gastrointestinal stromal tumors. Oncogene 26: 3909-3919, 2007.

31. Oliveira MJ, Costa AM, Costa AC, Ferreira RM, Sampaio P, Machado JC, Seruca R, Mareel M and Figueiredo C: CagA associates with c-met, E-cadherin and p120-catenin in a multiproteic complex that suppresses Helicobacter pylori-induced cell-invasive phenotype. J Infect Dis 200: 745-755, 2009. 